\title{
SOME EXTENSIONS OF APPROXIMATE CONTROLLABILITY RESULTS TO INVERSE PROBLEMS
}

\author{
AXEL OSSES AND JEAN-PIERRE PUEL
}

\begin{abstract}
We study here two inverse problems for the Laplace equation and for a generalized Stokes system, using approximate controllability methods. This enables us to give constructive results. The method is then used to develop numerical algorithms. Numerical results are given for the case of Laplace equation.
\end{abstract}

Key words: Laplace Equation, Stokes System, Inverse Problems, Approximate Controllability, Duality, Minimization Problem.

\section{INTRODUCTION}

We present here some extensions of a method which has been developped in [3] for the study of approximate controllability in semilinear heat equations. We consider here some elliptic problems, namely the Laplace equation or a generalized Stokes system for which, for example, the boundary value is unknown on a part of the boundary but some "measurements" of the solution are given on an internal surface. The problem is then to retrieve (an approximation of) the boundary value from the given measurements. This can be viewed as an inverse problem for which approximate controllability techniques apply and give a constructive method which can be used for numerical algorithms and effective computations.

In section 2 we consider the case of the Laplace equation and we give a precise description of the method.

In section 3, we present the numerical experiments and results while in section 4 we consider the case of a generalized Stokes system for which numerical calculations are in progress.

Our results make an extensive use of unique continuation properties which are classical for the case of Laplace equations but rely on new results by $\mathrm{C}$. Fabre and G. Lebeau ([1],[2]) in the case of Stokes systems.

\section{INVERSE PROBLEM FOR THE LAPLACE EQUATION}

Let $\Omega$ be a bounded regular open set of $\Re^{N}$. We denote by $\Gamma$ the boundary of $\Omega$, and by $\nu$ the unit exterior normal vector at a point of $\Gamma$. We suppose that $\Gamma=\bar{\Gamma}_{0} \cup \bar{\Gamma}_{1}$, where $\Gamma_{0}$ and $\Gamma_{1}$ are nonempty and relatively open.

For any $v \in L^{2}\left(\Gamma_{0}\right)$ we know that there exists a unique solution $y=y(v)$ of

$$
\begin{cases}-\Delta y=f & \text { in } \Omega, \\ y=v & \text { on } \Gamma_{0}, \\ y=0 & \text { on } \Gamma_{1},\end{cases}
$$

where $f$ is a given function in $L^{2}(\Omega)$.

REMARK 1. Problem (1) is solved by a transposition method (see [7]) and we only obtain $y \in L^{2}(\Omega)$ and $\Delta y \in L^{2}(\Omega)$. Nevertheless, it is classical to show that if $U$ is a neighborhood of $\Gamma_{0}, y$ is regular in $\Omega-U$. 


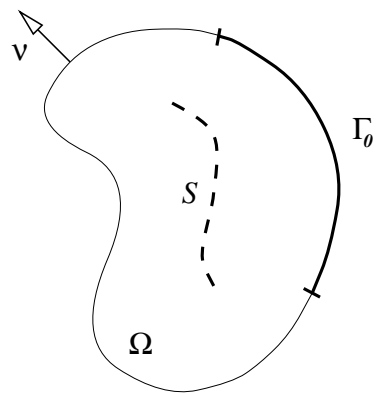

Figure 1. Domain $\Omega$ and curve $S$.

We are now given an internal regular surface $S$ (of codimension 1) such that $\bar{S} \subset \Omega$. From Remark 1 , the trace of $y(v)$ on $S$ has a perfect meaning in $H^{1 / 2}(S)$. Given $y_{1} \in L^{2}(S)$ and $\alpha>0$, our problem is now to look for $v \in L^{2}\left(\Gamma_{0}\right)$ such that

$$
\left|y(v)_{/ S}-y_{1}\right|_{L^{2}(S)} \leq \alpha
$$

REMARK 2. 1) $y_{1}$ represents some "measurements" of $y(v)_{/ S}$ and $v=y(v) / \Gamma_{0}$ is "unknown". We then try to find $v$ such that $y(v)_{/ S}$ is "almost" equal to the measurements.

2) Of course $\alpha=0$ would be optimal but the problem is then in general impossible as can be seen by regularity reasons for example. Take $f=0$ then $y(v)$ is analytic in $\Omega$ and $y_{1}$ is only taken in $L^{2}(S)$.

3) Our approach is inspired by approximate controllability methods as our problem is equivalent to showing that the set $\left\{y(v)_{/ S}, v \in L^{2}\left(\Gamma_{0}\right)\right\}$ is dense in $L^{2}(S)$.

Another classical approach (corresponding to an optimal control philosophy) for solving the inverse problem is to minimize

$$
H(v)=\frac{1}{2} \int_{S}\left|y(v)_{S}-y_{1}\right|^{2} d s+\beta \int_{\Gamma_{0}}|v|^{2} d \gamma, \beta>0
$$

The two methods present some similarities but are different. We will not consider the second one here.

In order to state our results, we have to make a geometrical hypothesis on $S$. Let us define

$$
\mathcal{C}=\{c \in C([0,1] ; \bar{\Omega}), \forall t \in] 0,1\left[, c(t) \in \Omega-\bar{S}, c(0) \in S, c(1) \in \Gamma_{0}\right\}
$$

The geometrical condition on $S$ can be stated as follows

$$
\bar{S} \in \Omega \text { and } \forall x \in S, \exists c_{x} \in \mathcal{C}, c_{x}(0)=x .
$$

Let us now define

$$
\begin{gathered}
S_{\text {ext }}=\{x \in \Omega-\bar{S}, \exists c \in \mathcal{C}, \exists t \in] 0,1[, c(t)=x\}, \\
S_{\text {int }}=\Omega-S_{\text {ext }}^{-}, \\
\Gamma_{00}=\left\{x \in \Gamma_{0}, \exists c \in \mathcal{C}, c(1)=x\right\} .
\end{gathered}
$$

We can easily prove the following properties

Proposition 3. (i) $\Gamma_{00}$ is a nonempty open subset of $\Gamma_{0}$ and without loss of generality we can assume that $\Gamma_{0}=\Gamma_{00}$ (by taking $v=0$ on $\left.\Gamma_{0}-\Gamma_{00}\right)$. 


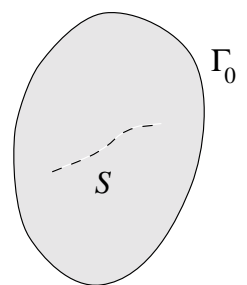

(a)

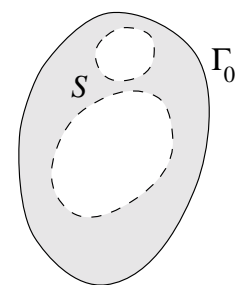

(b)

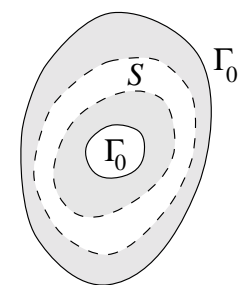

(c)

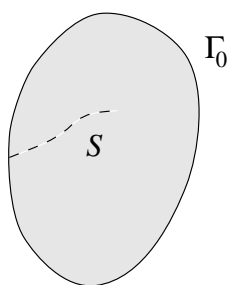

(d)

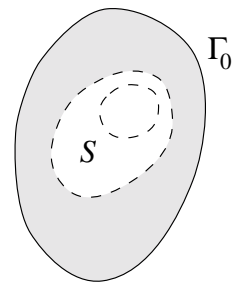

(e)

Figure 2. In cases (a), (b), (c), (3) is satisfied and in cases (d), (e), (3) is not satisfied.

(ii) $S_{e x t}$ is a nonempty open set and we have $: S \subset \partial S_{e x t}, \Gamma_{00} \subset \partial S_{e x t}$. Moreover, if $S_{\text {ext }}$ has several connected components, the boundary of each of them contains a nonempty open subset of $\Gamma_{00}$.

(iii) $S_{\text {int }}$ may be empty but if not, $\partial S_{\text {int }} \subset S \cup \Gamma$.

The following figure shows cases where (3) is satisfied and cases where (3) is not satisfied.

TheOREM 4. Let us assume that $S$ satisfies condition (3). Then for every $y_{1} \in L^{2}(S)$ and any $\alpha>0$, there exists $v \in L^{2}\left(\Gamma_{0}\right)$ such that

$$
\left|y(v)_{/ S}-y_{1}\right|_{L^{2}(S)} \leq \alpha \text {. }
$$

Moreover, if

$$
\begin{cases}-\Delta \bar{y}=f & \text { in } \Omega \\ \bar{y}=0 & \text { on } \Gamma\end{cases}
$$

we can take $v=-\frac{\partial \hat{\phi}}{\partial \nu} / \Gamma_{0}$ where (denoting by $\delta_{S}$ the Dirac mass on the surface S)

$$
\left\{\begin{array}{l}
-\Delta \hat{\phi}=\hat{\phi}_{0} \cdot \delta_{S} \text { in } \Omega \\
\hat{\phi}_{/ \Gamma}=0
\end{array}\right.
$$

and $\hat{\phi}_{0}$ minimizes over $L^{2}(S)$ the functional

$$
J\left(\phi_{0}\right)=\frac{1}{2} \int_{\Gamma_{0}}\left|\frac{\partial \phi}{\partial \nu}\right|^{2} d \gamma+\alpha\left|\phi_{0}\right|_{L^{2}(S)}-\int_{S}\left(y_{1}-\bar{y}_{/ S}\right) \phi_{0} d s .
$$

with

$$
\left\{\begin{array}{l}
-\Delta \phi=\phi_{0} . \delta_{S} \quad \text { in } \Omega \\
\phi_{/ \Gamma}=0
\end{array}\right.
$$

Proof. It can be shown directly, using Hahn Banach theorem, that $\{y(v) / s$, $\left.v \in L^{2}\left(\Gamma_{0}\right)\right\}$ is dense in $L^{2}(S)$ but we will give a proof using a duality argument as it is essential for deriving a constructive method. 
Take $\phi_{0} \in L^{2}(S)$ and $J\left(\phi_{0}\right)$ defined by (4). Notice that after translation by $\bar{y}$ we can assume that $f=0$. It is easy to show that $J$ is strictly convex and continuous on $L^{2}(S)$. Moreover one can show the following

Lemma 5. The functional $J$ is coercive if and only if the following unique continuation property holds.

$$
\begin{cases}-\Delta \phi=\phi_{0} \cdot \delta_{S} & \text { in } \Omega \\ \phi=0 & \text { on } \Gamma \\ \frac{\partial \phi}{\partial \nu}=0 & \text { on } \Gamma_{0}\end{cases}
$$

implies

$$
\phi=0 \text { in } \Omega \text { and } \phi_{0}=0
$$

Let us now show that when $S$ satisfies condition (3), the unique continuation property holds true. We have from (6)

$$
\begin{cases}-\Delta \phi=0 & \text { in } S_{e x t} \\ \phi=0 & \text { on } \Gamma_{00} \\ \frac{\partial \phi}{\partial \nu}=0 & \text { on } \Gamma_{00}\end{cases}
$$

Then, by classical unique continuation property for elliptic equations (see for example [11] for a general case and the bibliography therein), $\phi=0$ in $S_{e x t}$. Then $\phi=0$ on $\partial S_{e x t}$ and therefore $\phi=0$ on $S$. Now we have

$$
\left\{\begin{array}{l}
-\Delta \phi=0 \quad \text { in } S_{i n t} \\
\phi / \partial S_{i n t}=0
\end{array}\right.
$$

so that $\phi=0$ on $S_{\text {int }}$, and therefore $\phi=0$ in $\Omega$ and $\phi_{0}=0$, which shows the unique continuation property.

From the previous lemma we know that $J$ is coercive. Then $J$ has a unique minimum $\hat{\phi}_{0}$. It is easy to show that $\hat{\phi}_{0} \neq 0 \Leftrightarrow\left|y_{1}-\bar{y}_{/ S}\right|_{L^{2}(S)}>\alpha$, the case $\left|y_{1}-\bar{y}_{/ S}\right|_{L^{2}(S)} \leq \alpha$ being trivial as $v=0$ is solution to our problem. In the case $\left|y_{1}-\bar{y}_{/ S}\right|_{L^{2}(S)}>\alpha$, we obtain

$$
\left(J^{\prime}\left(\hat{\phi}_{0}\right), \theta_{0}\right)=0, \forall \theta_{0} \in L^{2}(S),
$$

so that, if $\theta$ denotes the solution of (5) associated with $\theta_{0}$, we have

$$
\forall \theta_{0} \in L^{2}(S), \int_{\Gamma_{0}} \frac{\partial \hat{\phi}}{\partial \nu} \frac{\partial \theta}{\partial \nu} d \gamma+\alpha \int_{S} \frac{\hat{\phi}_{0}}{\left|\hat{\phi}_{0}\right|_{L^{2}(S)}} . \theta_{0} d s-\int_{S}\left(y_{1}-\bar{y}_{/ S}\right) \theta_{0} d s=0
$$

Take $\hat{v}=-\frac{\partial \hat{\phi}}{\partial \nu} / \Gamma_{0}$ and $\hat{y}=y(\hat{v})$. we obtain

$$
\int_{\Gamma_{0}} \frac{\partial \hat{\phi}}{\partial \nu} \frac{\partial \theta}{\partial \nu} d \gamma=\int_{S}\left(\hat{y}_{/ S}-\bar{y}_{/ S}\right) \theta_{0} d s
$$

Therefore,

$$
\forall \theta_{0} \in L^{2}(S), \int_{S}\left(\hat{y}_{/ S}-y_{1}\right) \theta_{0} d s=-\alpha \int_{S} \frac{\hat{\phi}_{0}}{\left|\hat{\phi}_{0}\right|_{L^{2}(S)}} \cdot \theta_{0} d s
$$

which shows that

$$
\hat{y}_{/ S}-y_{1}=-\alpha \frac{\hat{\phi}_{0}}{\left|\hat{\phi}_{0}\right|_{L^{2}(S)}}
$$

and

$$
\left|\hat{y}_{/ S}-y_{1}\right|_{L^{2}(S)} \leq \alpha
$$




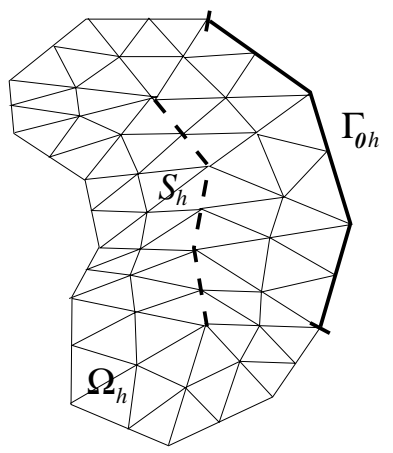

Figure 3.

REMARK 6. 1) As it was shown in $[4,5,6]$, by a duality argument, one can show that $\hat{v}=-\frac{\partial \hat{\phi}}{\partial \nu} / \Gamma_{0}$ is such that

$$
|\hat{v}|_{L^{2}\left(\Gamma_{0}\right)}=\operatorname{Min}\left\{|v|_{L^{2}\left(\Gamma_{0}\right)} ;\left|y(v)_{/ S}-y_{1}\right|_{L^{2}(S)} \leq \alpha\right\} .
$$

By considering a different functional $J$ as in [3], we could find a control $\tilde{v}$ minimizing the $L^{\infty}$ norm among admissible controls.

2) We can extend the method to a general second order elliptic operator such that the adjoint operator is coercive and regular.

3) Using a fixed point argument, we can extend the result to a semilinear problem of the form

$$
\begin{cases}-\Delta y+g(y)=f & \text { in } \Omega \\ y=v & \text { on } \Gamma_{0}, \\ y=0 & \text { on } \Gamma_{1},\end{cases}
$$

looking for $v \in L^{2}\left(\Gamma_{0}\right)$ such that $\left|y(v)_{/ S}-y_{1}\right|_{L^{2}(S)} \leq \alpha$, when $g$ is a non decreasing Lipschitz function. If we take $g(y)=|y|^{p-1} y$ with $p>1$, we can show that the result of approximate controllability is no more valid.

4) The detailed proofs and various extensions will be given in [8].

\section{Numerical Method and Experiments}

We take here for $\Omega$ a polygonal domain of $\Re^{2}$, for $S$ a polygonal internal curve in $\Omega$ and $f=0$. We consider a triangulation $\mathcal{T}_{h}$ of $\Omega$ such that $S$ and $\Gamma_{0}$ are union of triangles of $\mathcal{T}_{h}$, as in Figure 3 below.

We suppose that $\Gamma_{0}$ consists of $(Q-1)$ edges, $S$ consists of $(M-1)$ edges and $N$ is the total number of interior vertices in $\Omega$. Let

$$
V_{0 h}=\left\{\phi \in C^{0}(\bar{\Omega}), \forall T \in \mathcal{T}_{h}, \phi_{/ T} \in P_{1}, \phi_{/ \Gamma}=0\right\}
$$

and let $w_{1}, \ldots, w_{N}$ be a basis of $V_{0 h}$. We denote by $L_{h}$ a finite dimensional subspace of $L^{2}(S)$ consisting of piecewise constant functions on each edge of a triangle $T \in \mathcal{T}_{h}$ contained in $S$ (we could also take piecewise linear functions), and by $\phi_{1}, \ldots, \phi_{M}$ a basis of $L_{h}$.

If $\Phi \in L_{h}$, we call $T_{h} \Phi$ the solution of

$$
\left\{\begin{array}{l}
T_{h} \Phi \in V_{0 h}, \\
\int_{\Omega} \nabla T_{h} \Phi \nabla \psi d x=\int_{S} \Phi \psi d s, \quad \forall \psi \in V_{0 h} .
\end{array}\right.
$$


We consider the matrices

$$
\begin{gathered}
G_{k l}=\int_{\Gamma_{0}} \frac{\partial T_{h} \phi_{k}}{\partial \nu} \frac{\partial T_{h} \phi_{l}}{\partial \nu} d \gamma \\
C_{k l}=\int_{S} \phi_{k} \phi_{l} d s \\
f_{1 k}=\int_{S} y_{1} \phi_{k} d s .
\end{gathered}
$$

If $\Phi \in L_{h}$ we have $\Phi=\sum_{k=1}^{M} \beta_{k} \phi_{k}$ and if $\beta^{t}=\left(\beta_{1}, \ldots, \beta_{M}\right)$, we consider the functional

$$
J_{h}(\beta)=\frac{1}{2} \beta^{t} G \beta-\beta^{t} f_{1}+\alpha\left(\beta^{t} C \beta\right)^{1 / 2}
$$

and the minimization problem

$$
J_{h}(\hat{\beta})=\min _{\beta \in \Re^{M}} J_{h}(\beta) .
$$

Then, if $\hat{\Phi}_{h}=\sum_{k=1}^{M} \hat{\beta}_{k} \phi_{k}$, the approximate control is given by

$$
\hat{v}_{h}=-\frac{\partial T_{h} \hat{\Phi}_{h}}{\partial \nu}=-\sum_{k=1}^{M} \hat{\beta}_{k} \frac{\partial T_{h} \phi_{k}}{\partial \nu} .
$$

The numerical problem consists in solving $M$ problems (9) with right hand sides $\phi_{k}$ and then in minimizing $J_{h}$.

REMARK 7. 1) The problem of computing the normal derivatives is not simple and may generate large errors. Various choices can be made for this computation.

2) Matrix $G$ is badly conditionned so we use a preconditionner $\beta=$ $\operatorname{diag}\left(G_{i i}^{-1 / 2}\right) \beta^{\prime}$ and we work with double precision variables.

The following computations have been made using MODULEF for the mesh generation and elementary matrices, a Cholesky decomposition for solving the $N \times N$ linear systems (with $M$ right hand sides) and the BFGS method (see [10]) for the minimization problem.

In Figure 4 we present three numerical experiments corresponding to different positions of $\Gamma_{0}, S$ and different $\Omega$. For all these three cases we have taken $y_{1}=1$ on $S$. The control $\hat{v}_{h}$ is schematically represented normally to $\Gamma_{0}$. The value of the solution $\hat{y}_{h}$ is represented by levels of grey and the white region near each curve $S$ corresponds to the level $1.00 \pm 0.03$.

On Figure 5 we consider two other functions $y_{1}$ in the geometry of first case in Figure 4 : a sine function and a Heaviside function. There is an important error for the case of Heaviside function and this was expected as the solution $\hat{y}$ must be very regular on $S$.

Figure 6 shows the relation between the relative theoretical and numerical errors and $\alpha$ in the first example of Figure 4 (the results for all examples are similar), where the relative theoretical error is

$$
\frac{\left|\hat{y}-y_{1}\right|_{L^{2}(S)}}{\left|y_{1}\right|_{L^{2}(S)}}=\left\{\begin{array}{lll}
\frac{\alpha}{\sqrt{\left.y_{1}\right|_{L^{2}(S)}}} & \text { if } & \alpha<\left|y_{1}\right|_{L^{2}(S)} \\
1 & \text { if } & \alpha \geq\left|y_{1}\right|_{L^{2}(S)}
\end{array}\right.
$$

and the relative numerical error is

$$
e(\alpha)=\frac{\left|\hat{y}_{h}-y_{1}\right|_{L^{2}(S)}}{\left|y_{1}\right|_{L^{2}(S)}} .
$$



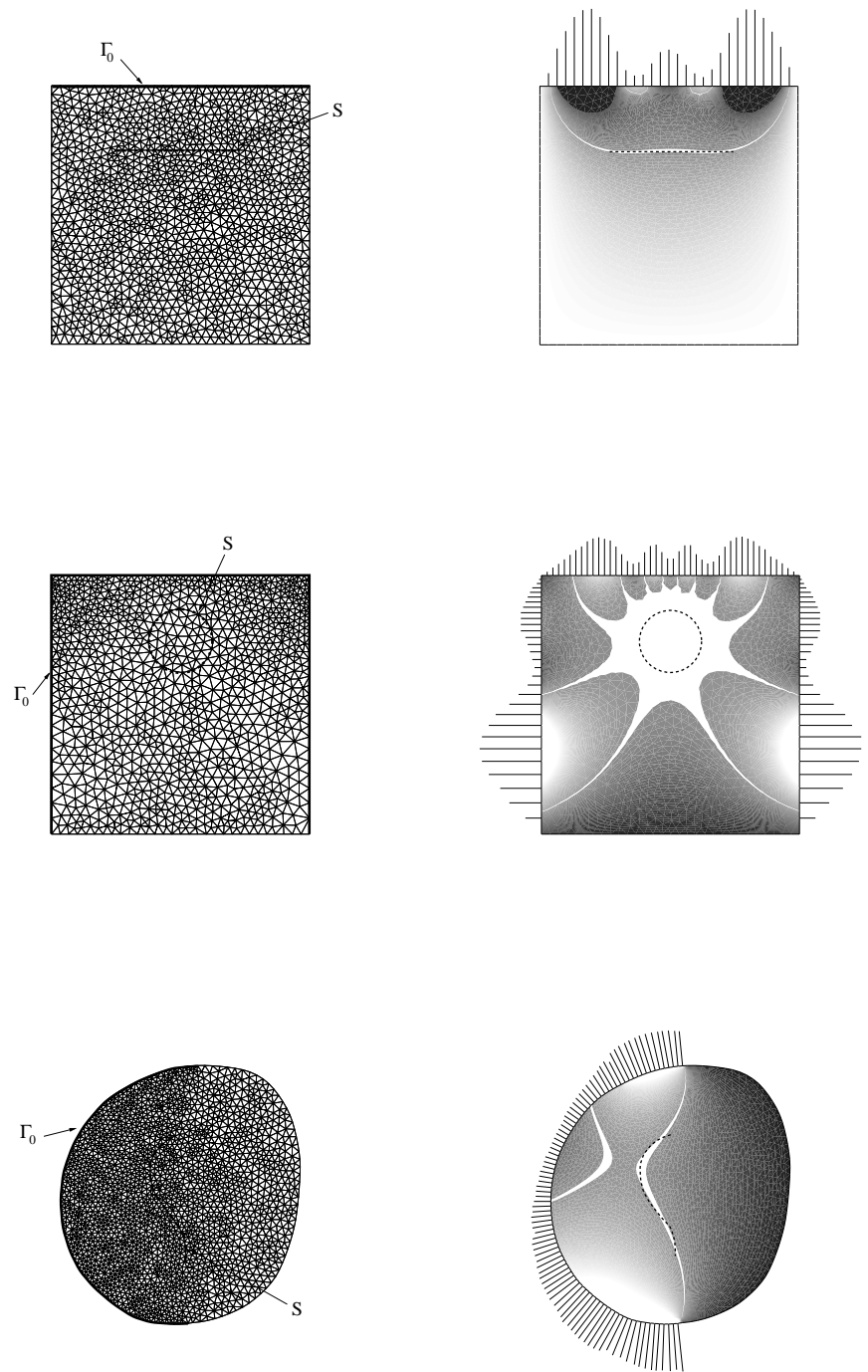

Figure 4.

These results show that a mesh refinement does not improve the precision below a critical value of $\alpha$. We don't have a precise explanation of this phenomenon at the moment.

\section{Inverse Problem for a Generalized Stokes System}

We keep the same notations as in Section 2 and we consider the standard functional spaces

$$
\begin{gathered}
H=\left\{z \in\left(L^{2}(\Omega)\right)^{N}, \operatorname{div} z=0\right\} \\
V=\left\{y \in\left(H_{0}^{1}(\Omega)\right)^{N}, \operatorname{div} y=0\right\} \\
L_{0}^{2}\left(\Gamma_{0}\right)=\left\{v \in\left(L^{2}\left(\Gamma_{0}\right)\right)^{N}, \int_{\Gamma_{0}} v \cdot \nu d \gamma=0\right\}
\end{gathered}
$$



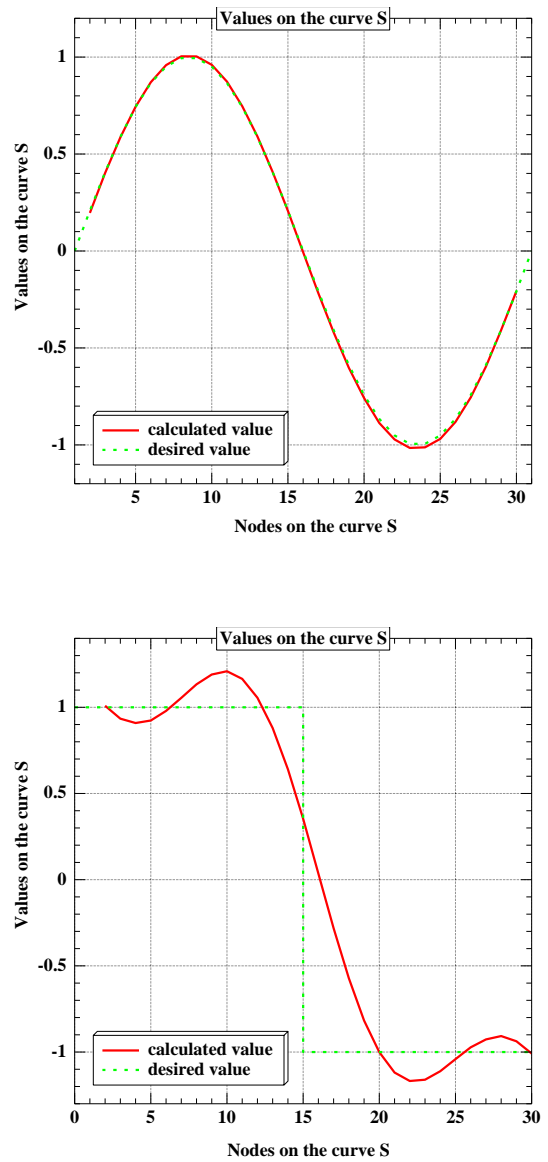

Figure 5.

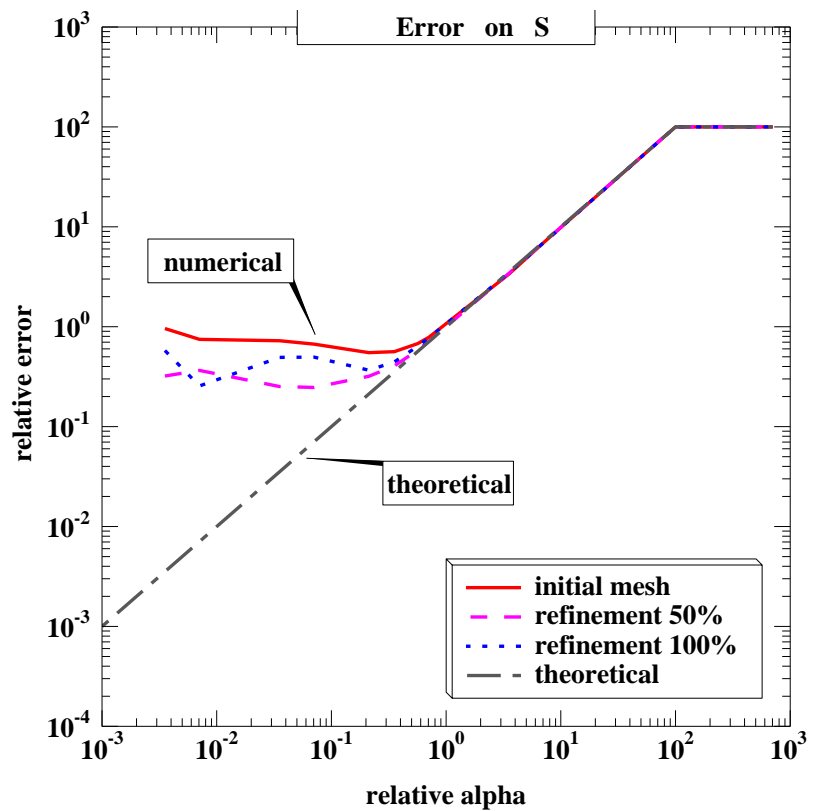

Figure 6. 
Let us take $a \in\left(L^{\infty}(\Omega)\right)^{N}$, diva $=0$ and $f \in\left(L^{2}(\Omega)\right)^{N}$. For $v \in L_{0}^{2}\left(\Gamma_{0}\right)$ we consider the following problem

$$
\left\{\begin{array}{l}
-\Delta y_{i}+\sum_{j=1}^{N} \frac{\partial}{\partial x_{j}}\left(a_{j} y_{j}\right)=f_{i}-\frac{\partial p}{\partial x_{i}} \quad \text { in } \Omega, \quad i=1, \ldots, N \\
\operatorname{div} y=0, \\
y_{/ \Gamma_{0}}=v, \\
y_{/ \Gamma_{1}}=0 .
\end{array}\right.
$$

Proposition 8. There exists a unique solution $(y, p)=(y(v), p(v))$ to (12) with $y \in H, p \in L_{l o c}^{2}(\Omega) / \Re$. The correct definition of $y$ is given by the transposition method as follows :

for every $h \in\left(L^{2}(\Omega)\right)^{N}$, let $(\psi, q) \in V \times L^{2}(\Omega)_{/ \Re}$ be solution of

$$
\left\{\begin{array}{l}
-\Delta \psi-(a . \nabla) \psi=h-\nabla q \quad \text { in } \Omega, \\
\operatorname{div} \psi=0, \\
\psi / \Gamma=0 .
\end{array}\right.
$$

Then $(\psi, q) \in\left(H^{2}(\Omega)\right)^{N} \times H^{1}(\Omega)$ (see [12]) and we have

$$
\forall h \in\left(L^{2}(\Omega)\right)^{N}, \int_{\Omega} y \cdot h d x=\int_{\Omega} f \cdot \psi d x-\int_{\Gamma_{0}} v \cdot\left(\frac{\partial \psi}{\partial \nu}-q \nu\right) d \gamma
$$

Remark 9. Condition diva $=0$ is only required for having an existence result in (13)

The result of Proposition 8 comes from the classical application of the transposition method. Interpretation of (14) gives (12) except for the boundary condition which does not make full sense for $y \in H$. We completely recover the normal component of the boundary condition whereas the tangential components are obtained only if $y$ is more regular. Anyway, there exists a unique solution to (14) and we can show that if $U$ is a neighborhood of $\Gamma_{0}$, then $y \in\left(H^{1}(\Omega-U)\right)^{N}$ so that if $S$ is an internal surface such that $\bar{S} \subset \Omega$, the trace $y_{/ S}$ makes perfect sense. Our problem is then the following : Given $y_{1} \in\left(L^{2}(S)\right)^{N}$ and $\alpha>0$, find $v \in L_{0}^{2}\left(\Gamma_{0}\right)$ such that

$$
\left|y(v)_{/ S}-y_{1}\right|_{\left(L^{2}(S)\right)^{N}} \leq \alpha .
$$

THEOREM 10. If $S$ satisfies the geometrical condition (3) then for every $y_{1} \in\left(L^{2}(S)\right)^{N}$ and every $\alpha>0$, there exists $v \in L_{0}^{2}\left(\Gamma_{0}\right)$ such that

$$
\left|y(v)_{/ S}-y_{1}\right|_{\left(L^{2}(S)\right)^{N}} \leq \alpha .
$$

Proof. We only sketch the proof here as we use the same method as in Section 2 and for simplicity we will take $f=0$.

Given $\phi_{0} \in\left(L^{2}(S)\right)^{N}$, there exists a unique couple $(\phi, \pi) \in V \times L^{2}(\Omega)_{/ \Re}$ such that

$$
\left\{\begin{array}{l}
-\Delta \phi-(a . \nabla) \phi=\phi_{0} . \delta_{S}-\nabla \pi \quad \text { in } \Omega \\
\operatorname{div} \phi=0 \\
\phi_{/ \Gamma}=0 .
\end{array}\right.
$$

We can show that if $\omega$ is an open subset of $\Omega$ such that $\omega \cap \bar{S}=\emptyset$, then $\phi \epsilon$ $\left(H^{2}(\omega)\right)^{N}$ and $\pi \in H^{1}(\omega)$, so that $\phi$ and $\pi$ are "regular" in the neighborhood of $\Gamma_{0}$.

Denoting by $P_{0}$ the orthogonal projection from $\left(L^{2}\left(\Gamma_{0}\right)\right)^{N}$ onto $L_{0}^{2}\left(\Gamma_{0}\right)$ we define

$$
J\left(\phi_{0}\right)=\frac{1}{2} \int_{\Gamma_{0}}\left|P_{0}\left(\frac{\partial \phi}{\partial \nu}-\pi \nu\right)\right|^{2} d \gamma+\alpha\left|\phi_{0}\right|_{\left(L^{2}(S)\right)^{N}}-\int_{S} y_{1} \cdot \phi_{0} d s .
$$


Then $J$ is strictly convex and continuous and again the coercivity of $J$ is equivalent to the following unique continuation property :

Let $(\phi, \pi)$ be solution of $(15)$ such that

$$
P_{0}\left(\frac{\partial \phi}{\partial \nu}-\pi \nu\right)=0 \text { on } \Gamma_{0}
$$

Then $\phi=0, \pi=C$ st in $\Omega$ so that $\phi_{0}=0$. The main difficulty is then to prove the unique continuation property with $a \in\left(L^{\infty}(\Omega)\right)^{N}$ only. We have (by adding a suitable constant to $\pi$ )

$$
\begin{cases}-\Delta \phi-(a . \nabla) \phi=-\nabla \pi & \text { in } S_{e x t}, \\ \operatorname{div} \phi=0, & \text { in } S_{e x t}, \\ \phi_{/ \Gamma_{0}}=0,\left(\frac{\partial \phi}{\partial \nu}-\pi \nu\right) / \Gamma_{0}=0 . & \end{cases}
$$

From a result by C. Fabre and G. Lebeau (see [2]) which relies on new ad'hoc Carleman inequalities, we know that $\phi=0$ in $S_{e x t}$ and therefore $\phi=0$ on $S$. Now in $S_{\text {int }}$ we have a homogeneous Stokes system with zero right hand side which shows that $\phi=0$ in $S_{\text {int }}$. Therefore, $\phi=0$ and $\pi=C$ st in $\Omega$, so that $\phi_{0}=0$.

As a consequence, there exists a unique $\hat{\phi}_{0} \in\left(L^{2}(S)\right)^{N}$ such that

$$
J\left(\hat{\phi}_{0}\right)=\min _{\phi_{0} \in\left(L^{2}(S)\right)^{N}} J\left(\phi_{0}\right)
$$

If we write $(\hat{\phi}, \hat{\pi})$ the solution of $(15)$ associated with $\hat{\phi}_{0}, \hat{v}=-P_{0}\left(\frac{\partial \hat{\phi}}{\partial \nu}-\hat{\pi} \nu\right)$ and $\hat{y}=y(\hat{v})$, it is easy to show that

$$
\left|\hat{y}_{/ S}-y_{1}\right|_{\left(L^{2}(S)\right)^{N}} \leq \alpha .
$$

REMARK 11. It is possible to extend the result to some nonlinear systems by using a fixed point argument, taking $a=a(y)$. But we need $a$ to be bounded in $\left(L^{\infty}(\Omega)\right)^{N}$ and to satisfy diva $=0$. So it seems difficult to obtain relevant nonlinearities. For the time dependent problem, the condition diva $=0$ is no more necessary and we can obtain a wider class of nonlinearities. Unfortunately the case of Navier Stokes equations cannot be treated up to now.

As for the case of Laplace equation, the method leads to numerical algorithms. The numerical results will be presented together with a complete version of our work in [9].

\section{REFERENCES}

[1] C.FABRE. Uniqueness results for general Stokes equations and their consequences in control problems for linear and nonlinear equations. To appear in ESAIM : Control, Optimisation and Calculus of Variations.

[2] C.Fabre and G.Lebeau. Prolongement unique des solutions de l'équation de Stokes. To appear in Comm. in P.D.E.

[3] C.Fabre, J.-P.Puel, E.Zunzua. Approximate controllability of the semilinear heat equation. Proc. of the Royal Soc. of Edinburgh, 125A, p. 31-61 (1995)

[4] J.-L.LIONS. Exact controllability, stabilization and perturbations for distributed systems. SIAM Rev., 30, p. 1-68 (1988).

[5] J.-L.Lions. Remarques sur la contrôlabilité approchée. Proceedings of "Jornadas Hispano Francesas sobre control de sistemas distribuidos", University of Malaga, Spain (1990).

[6] J.-L.Lions. Remarks on approximate controllability. Israel J. of Math. (1992).

[7] J.-L.Lions and E.Magenes. Problèmes aux limites non homogènes et applications. Vol. 1, Dunod (1968). 
[8] A.Osses and J.-P.PuEL. On the controllability of the semilinear Laplace equation on an interior curve. To appear.

[9] A.Osses and J.-P.PuEL. Contrôlabilité sur une courbe intérieure pour l'équation de Stokes stationnaire avec un potentiel. To appear.

[10] W.Press, S.Teukolsky, W.Vetterling, B.Flannery. Numerical recipes in $C$, the art of scientific computing. Cambridge, Cambridge University Press (1992).

[11] J.-C.SAUT and B.ScheURER. Sur l'unicité du problème de Cauchy et le prolongement unique des équations elliptiques à coefficients non localement bornés. Journal of Differential Equations 43, p. 28-43, (1982).

[12] R.Temam. Navier Stokes Equations. North Holland, Amsterdam (1977).

Centre de Mathématiques Appliquées, Ecole Polytechnique 91128 Palaiseau CEDEX

Université de Versailles Saint-Quentin et Centre de Mathématiques Appliquées, Ecole Polytechnique 91128 Palaiseau Cedex 